\title{
ARTICLE
}

\section{Leptin prevents the metabolic effects of adiponectin in L6 myotubes}

\author{
X. Fang • J. Fetros • K. E. Dadson • A. Xu • G. Sweeney
}

Received: 2 May 2009 /Accepted: 22 June 2009 /Published online: 28 July 2009

(C) Springer-Verlag 2009

\begin{abstract}
Aims/hypothesis Adiponectin and leptin are negatively and positively correlated with human obesity respectively, and have both been shown to regulate energy metabolism in skeletal muscle. However, little is known about their signalling and functional crosstalk. Here we investigated the effects of leptin on metabolic actions of (1) globular adiponectin (gAd) and (2) full-length adiponectin (fAd) in L6 cells.

Methods Glucose uptake was measured upon gAd and fAd treatment after incubation with different doses $(0.3,0.6,3$, $6,60 \mathrm{nmol} / \mathrm{l}$ ) of leptin for 6,12 and $24 \mathrm{~h}$. We also measured adiponectin receptor (ADIPOR) expression and stimulation of downstream signalling by $\mathrm{gAd}$ and fAd using coimmunoprecipitation and western blotting following leptin pretreatment, as well as analysis of fatty acid uptake and oxidation using radiolabelled tracers.

Results Leptin attenuated the stimulation of glucose uptake by gAd and fAd in a dose- and time-dependent manner, a finding correlated with decreased levels of ADIPOR1 and ADIPOR2. gAd and fAd increased palmitate uptake via
\end{abstract}

X. Fang $\cdot$ J. Fetros $\cdot$ K. E. Dadson $\cdot$ G. Sweeney $(\bowtie)$

Department of Biology, York University,

4700 Keele St, Toronto,

M3J 1P3 Toronto, ON, Canada

e-mail: gsweeney@yorku.ca

\section{A. $\mathrm{Xu}$}

Department of Medicine and Department of Pharmacology,

University of Hong Kong,

Hong Kong, People's Republic of China

G. Sweeney

Institut Pasteur Korea,

Seoul, South Korea activation of AMP protein kinase (T172), enhanced expression of the fatty acid transporter CD36, phosphorylated acetyl-CoA carboxylase (S79) and enhanced palmitate oxidation, all of which were attenuated by leptin pretreatment. Adiponectin can also enhance insulin sensitivity via direct signalling crosstalk; here we show that enhanced insulin-stimulated IRS-1 (Y612) and Akt (T308) phosphorylation in response to fAd was attenuated by leptin. APPL1 was recently identified as a critical mediator of adiponectin action in skeletal muscle. We demonstrated that leptin attenuated binding of APPL1 to LKB1, a downstream target leading to AMPK phosphorylation.

Conclusions/interpretation The direct metabolic and insulin-sensitising effects of adiponectin were attenuated in the presence of leptin.

Keywords ADIPOR - Adiponectin - AMPK . Insulin-sensitising effects $\cdot$ Leptin $\cdot$ Metabolism
Abbreviations
ACC Acetyl-CoA carboxylase
ADIPOR Adiponectin receptor
AICAR 5-Amino-4-imidazole carboxamide riboside
AMEM Alpha modification Eagle's medium
AMPK AMP protein kinase
APPL1 Adapter protein containing a pleckstrin homol- ogy domain, phosphotyrosine binding (PTB) domain and leucine zipper motif
fAd Full-length adiponectin
FATP Fatty acid transport protein
gAd Globular adiponectin
HEK Human embryonic kidney
LKB1 Protein serine/threonine kinase
siRNA Small interfering RNA 


\section{Introduction}

The prevalence of obesity has become a major health concern due to association with many complications such as type 2 diabetes and cardiovascular disease $[1,2]$. One potential underlying mechanism is altered endocrine effects of adipose-derived hormones [3]. Due to its anti-diabetic, anti-atherogenic, anti-inflammatory and cardioprotective functions [4, 5], adiponectin has attracted great interest since being identified. The metabolic effects of adiponectin, both direct and via insulin sensitisation, have been identified in skeletal muscle and liver by several groups $[6,7]$. Moreover, the synergistic effect of adiponectin and insulin on glucose uptake in L6 muscle cells was reported by our group [7]. It has also been demonstrated that adiponectin modulates carbohydrate and lipid metabolism in muscle primarily through stimulation of AMP protein kinase (AMPK) and peroxisome proliferator-activated receptor alpha [8]. More recently, Liu's group have shown that adiponectin and insulin signalling crosstalk via a mechanism involving the LKB1/AMPK/tuberous sclerosis $1 / 2$ pathway [9].

In circulation, adiponectin exists as multimers, namely low molecular weight trimers, middle molecular weight hexamers and high molecular weight oligomers [10]. The formation of these distinct structures is physiologically significant since oligomers have been observed to be capable of mediating distinct cellular effects [11]. Adiponectin can also be proteolytically cleaved to yield the globular C-terminal domain of adiponectin, which mediates potent metabolic effects in skeletal muscle [7, 12].

Adiponectin mediates its effects through at least two receptor isoforms, adiponectin receptor (ADIPOR) 1 and ADIPOR2 [8]. Decreased levels of ADIPORs were observed in many, but not all, studies involving obese and diabetic patients as well as animal models [13, 14]; these decreases can often be corrected by exercise [15]. ADIPORs were recently shown to interact with a novel adapter protein containing a pleckstrin homology domain, phosphotyrosine binding (PTB) domain and leucine zipper motif (APPL1). This binding mediated adiponectin effects in muscle and endothelial cells, and, importantly, was identified as a major point of crosstalk with insulin signalling [16-18].

Hyperleptinaemia is a characteristic feature in human obesity. Several recent studies have alluded to the potential crosstalk between physiological actions of leptin and adiponectin [19, 20]. Leptin is known to regulate a wide range of intracellular signalling pathways [21]; however the potential crosstalk between leptin and adiponectin signalling is unclear. To date, three recent studies examining cell cycle regulation and cell proliferation have demonstrated that adiponectin and leptin can crosstalk [22-24]. The objective of this study was to characterise the effect of chronic leptin pretreatment on subsequent adiponectin signalling and consequently on metabolic function in rat skeletal muscle cells.

\section{Methods}

Materials $\left[9,10-{ }^{3} \mathrm{H}\right]$ Palmitic acid and 2-deoxy-D- $\left[{ }^{3} \mathrm{H}\right]$ glucose were purchased from Amersham (Baie d'Urfé, QC, Canada). Recombinant murine leptin and compound $\mathrm{C}$ were purchased from Calbiochem (San Diego, CA, USA). Insulin (Humulin) was obtained from Eli Lilly (Toronto, ON, Canada). TRIzol reagent and Platinum SYBR Green qPCR SuperMix UDG Kit were obtained from Invitrogen Life Technologies (Burlington, ON, Canada). Anti-APPL1 antibody was produced in-house by immunisation of rabbits as previously described [16]. Horseradish peroxidase-linked anti-rabbit antibody, phospho-specific antibodies for rabbit AMPK (Thr 172), ACC (Ser 79), Akt (Thr 308), Akt and $\beta$-actin were purchased from Cell Signaling (Beverly, MA, USA). Fatty acid transport protein (FATP)1, FATP4, AMPK $\alpha 1$, AMPK $\alpha 2$, CD36, IRS-1 and LKB1 antibodies were obtained from Santa Cruz Biotechnology (Santa Cruz, CA, USA). Phospho-specific antibody for IRS-1(Tyr612) was obtained from BioSource (Camarillo, CA, USA). ADIPOR1 and ADIPOR2 antibodies were obtained from IBL (Takasaki, Japan). All other chemicals were purchased from Sigma-Aldrich Canada (Oakville, ON, Canada). All cell culture components were purchased from Wisent (StBruno, QC, Canada).

Production of recombinant gAd and fAd Recombinant globular adiponectin (gAd) was produced by subcloning murine gAd cDNA (a kind gift from P. Scherer, Department of Cell Biology, Bronx, Albert Einstein College of Medicine, Bronx, NY, USA) into the pTrisEx expression vector (Novagen, Gibbstown, NJ, USA) as described previously [25]. A human embryonic kidney (HEK) cell line stably expressing recombinant full-length multimers of adiponectin (fAd) was generated as previously described [26]. HEK cells were cultured in DMEM containing 10\% (vol./vol.) FBS and allowed to secrete adiponectin into serum-free alpha modification Eagle's medium (AMEM) for $24 \mathrm{~h}$. The conditioned medium was concentrated using stirred ultrafiltration cell system (Millipore, Billerica, MA, USA) with ultrafiltration membrane (NMWL $30 \mathrm{kDa}$; Millipore). Conditioned medium containing adiponectin was then diluted to $100 \mathrm{mg} / \mathrm{l}$ in serum-free AMEM and stored at $-80^{\circ} \mathrm{C}$. Control conditioned medium was made using HEK cell line transfected with vector without adiponectin sequence. Glucose uptake was performed to test biological activity of fAd and gAd. The concentrations and treatment time of fAd and gAd used in this study were 
based on preliminary studies and various publications by ourselves and others [7, 25].

Cell culture Rat L6 skeletal muscle cells were grown in AMEM containing $10 \%$ (vol./vol.) FBS under $5 \% \mathrm{CO}_{2}$ at $37^{\circ} \mathrm{C}$. For experimental procedures, myoblasts were cultured in 2\% FBS AMEM for 5 to 7 days for differentiation of myotubes.

Analysis of Adipor1 and -2 expression L6 myotubes were incubated with leptin $(60 \mathrm{nmol} / \mathrm{l})$ for $24 \mathrm{~h}$, then RNA was isolated. Adipor 1, Adipor 2 and $\beta$-actin expression levels were measured using platinum SYBR Green qPCR SuperMix UDG Kit. Primer sequences for the genes were: Adipor1: forward 5'-GCTGGCCTTTATGCTGCTCG-3', reverse 5'-TCTAGGCCGTAACGGAATTC-3'; Adipor2: forward 5'-CCCTCTGCAAGAGAAAGTGG-3', reverse 5'TAGCCAGCCTATCTGCCCTA-3'; $\beta$-actin: forward 5'-CTGTGCCCATCTATGAGGGT-3', reverse 5'-CTCTCA GCTTGGTGGTGAA-3'. The annealing temperature for Adipor 1 and Adipor 2 was $60^{\circ} \mathrm{C}$, that for $\beta$-actin was $65^{\circ} \mathrm{C}$; 40 cycles were performed.

Measurement of glucose uptake L6 myotubes were incubated for $24 \mathrm{~h}$ with different concentrations of leptin (0.3, $0.6,3,6,60 \mathrm{nmol} / \mathrm{l})$. For time-course experiments, cells were incubated with $6 \mathrm{nmol} / 1$ leptin for 6,12 and $24 \mathrm{~h}$. Cells were then treated for $2 \mathrm{~h}$ with $5 \mathrm{mg} / \mathrm{l} \mathrm{fAd}$ or gAd in the presence of leptin. After treatment, uptake of 2-deoxyD- $\left[{ }^{3} \mathrm{H}\right]$ glucose was determined as described previously [7]. Briefly, cells were incubated for $5 \mathrm{~min}$ at room temperature in transport solution. Cells were then lysed and transferred to scintillation vials for ${ }^{3} \mathrm{H}$ radioactivity counting. Values were expressed in pmol $\mathrm{min}^{-1}$ (mg protein) ${ }^{-1}$ (see results section) and expressed in graphs as fold of basal pmol $\min ^{-1}$ (mg protein $)^{-1}$.

Measurement of fatty acid uptake Cells were grown in 24well plates. Leptin was added to appropriate wells to a final concentration of $60 \mathrm{nmol} / 1$ and wells were incubated for $24 \mathrm{~h}$. Cells were serum-starved for $4 \mathrm{~h}$ and then treated with fAd $(5 \mu \mathrm{g} / \mathrm{ml})$ or gAd $(5 \mu \mathrm{g} / \mathrm{ml})$ in the presence or absence of AMPK inhibitor compound $\mathrm{C}(20 \mu \mathrm{mol} / \mathrm{l})$. $\left[9,10-{ }^{3} \mathrm{H}\right]$ Palmitic acid was measured as previously described [7, 27]. Briefly, cells were washed and treated for $1 \mathrm{~min}$ with transport solution (140 mmol/l NaCl, $20 \mathrm{mmol} / \mathrm{l}$ HEPES-Na, $2.5 \mathrm{mmol} / 1 \mathrm{MgSO}_{4}, 1 \mathrm{mmol} / \mathrm{l} \mathrm{CaCl}, 5 \mathrm{mmol} / 1 \mathrm{KCl}, 20$ $\mu \mathrm{mol} / 1$ sodium palmitate, $18.5 \mathrm{MBq} / 1\left[9,10{ }^{3} \mathrm{H}\right]$ palmitic acid, $\mathrm{pH}$ 7.4). Cells were lysed with $1 \mathrm{~mol} / \mathrm{l} \mathrm{KOH}$. Lysates were transferred to vials containing scintillation fluid for radioactive counting and used for protein determination. Values were expressed in pmol min ${ }^{-1}$ (mg protein) ${ }^{-1}$ and shown in graphs as fold of basal pmol $\min ^{-1}$ (mg protein $)^{-1}$.
Measurement of fatty acid oxidation Myotubes were cultivated in 12-well plates and incubated in the presence of leptin $(60 \mathrm{nmol} / \mathrm{l})$ for $24 \mathrm{~h}$. fAd and $\operatorname{gAd}(5 \mu \mathrm{g} / \mathrm{ml}$ each $)$ were added to cells under normal and leptin pretreatment conditions. 5-Amino-4-imidazole carboxamide riboside (AICAR) was added to a final concentration of $2 \mathrm{mmol} / \mathrm{l}$ as positive control. After $6 \mathrm{~h}$ incubation in AMEM containing $0.1 \mathrm{mmol} / 1$ palmitate $\left(9,10-\left[{ }^{3} \mathrm{H}\right]\right.$ palmitate, $37 \mathrm{MBq} / \mathrm{l})$ and $2 \%(\mathrm{wt} / \mathrm{wt}$ ) BSA, palmitate oxidation was assessed by measuring ${ }^{3} \mathrm{H}_{2} \mathrm{O}$ produced in the incubation medium as described previously [28]. Medium was collected into Eppendorf tubes. Then 10\% (vol./vol.) trichloroacetic acid was added to each tube and tubes were centrifuged for $10 \mathrm{~min}$ at $14,999 \mathrm{~g}$ at $4^{\circ} \mathrm{C}$. The supernatant fraction was collected into capless Eppendorf tubes. These tubes were placed in vials containing equal volumes of distilled water and incubated overnight in a $50^{\circ} \mathrm{C}$ water bath. After incubation, $5 \mathrm{ml}$ of scintillation liquid was added to each vial for radioactive counting; $1 \mathrm{~mol} / \mathrm{l} \mathrm{KOH}$ was added to prepare lysates and determine protein concentration. As previously published using this method, values are expressed in cpm/mg protein [28].

Small interfering RNA to knockdown expression of AMPK $\alpha$ L6 cells were cultured in 24-well tissue culture plates and transfected for $24 \mathrm{~h}$ with $50 \mathrm{nmol} / 1 A M P K \alpha 1$ (also known as PRKAA1) and $50 \mathrm{nmol} / 1$ AMPK $\alpha 2$ (also known as PRKAA2), or with scrambled small interfering RNA (siRNA) (Silencer Negative Control 1 siRNA; Ambion, Austin, TX, USA) as previously described [29]. siRNA sequences for $A M P K \alpha 1$ and $A M P K \alpha 2$ were: $A M P K \alpha 1$ GCAUAUGCUGCAGGUAGAU; AMPK $\alpha 2$, CGUCAUU GAUGAUGAGGCU (Ambion).

Western blot analysis L6 myotubes were cultured in sixwell plates and incubated with leptin $(60 \mathrm{nmol} / \mathrm{l})$ for $24 \mathrm{~h}$. Cells were serum-starved for $4 \mathrm{~h}$ before stimulation with $\operatorname{gAd}(5 \mu \mathrm{g} / \mathrm{ml})$ or fAd $(5 \mu \mathrm{g} / \mathrm{ml})$. Cell lysates were resolved by SDS-PAGE and transferred to a nitrocellulose membrane.

Co-immunoprecipitation Cells were incubated with leptin $(60 \mathrm{nmol} / \mathrm{l})$ for $24 \mathrm{~h}$ and treated with $\mathrm{gAd}(5 \mu \mathrm{g} / \mathrm{ml})$ or fAd $(5 \mu \mathrm{g} / \mathrm{ml})$. After treatment, cells were lysed with RIPA buffer (1\% (vol./vol.) NP40, 0.5\% (wt/wt) SDC, $0.1 \%$ (wt/ wt) SDS in PBS buffer, pH7.4) complemented with protease inhibitor mixture on ice. Non-soluble material was discarded after centrifugation $(16,249 \mathrm{~g}$ for $5 \mathrm{~min}$ at $4^{\circ} \mathrm{C}$ ) and the supernatant fraction was incubated overnight with $1 \mu \mathrm{g}$ of mouse monoclonal LKB1 antibody. Then protein A sepharose beads (GE Healthcare Bio-Sciences, Baie d'Urfé, QC, Canada) were added and incubated for $3 \mathrm{~h}$. After incubation, the beads were pelleted and washed. Beads were resuspended in SDS sample buffer, supple- 
mented with $10 \%$ (vol./vol.) $\beta$-mercaptoethanol, then boiled for $5 \mathrm{~min}$ and subjected to western blot analysis using polyclonal antibody against APPL1.

Statistical analysis Data are expressed as mean values \pm SEM with number of repeats $(n)$ stated in each case. Statistical analysis was undertaken using two-way ANOVA or the paired Student's $t$ test where appropriate. Differences between groups were considered statistically significant at $p<0.05$.

\section{Results}

Prevention of fAd-and gAd-stimulated glucose uptake by leptin in a time- and dose-dependent manner We evaluated the effect of varying leptin concentrations $(0.3,0.6,3,6$, $60 \mathrm{nmol} / \mathrm{l})$ and pretreatment times $(6,12,24 \mathrm{~h})$ on the ability of fAd or gAd to stimulate 2-deoxy-D- $\left[{ }^{3} \mathrm{H}\right]$ glucose uptake. The concentrations of leptin were chosen, on the basis of published literature by our own and other groups, as those used to elicit physiological effects in cultured cells $[30,31]$. Basal glucose uptake levels remained unchanged by all leptin concentrations (Fig. 1a, b). Both fAd $(5 \mu \mathrm{g} / \mathrm{ml}$, $2 \mathrm{~h})$ and $\mathrm{gAd}(5 \mu \mathrm{g} / \mathrm{ml}, 2 \mathrm{~h})$ elicited a statistically significant increase in glucose uptake under normal, 0.3 and $0.6 \mathrm{nmol} / 1$ leptin treatment conditions (Fig. 1a, b). Our data revealed that pretreatment of cells with leptin for $24 \mathrm{~h}$ inhibited fAd- or gAd-stimulated 2-deoxy-D- $\left[{ }^{3} \mathrm{H}\right]$ glucose uptake in a dose-dependent manner (Fig. 1a, b). A significant inhibitory effect was observed at 3, 6 and $60 \mathrm{nmol} / \mathrm{l}$ for fAd and gAd. When cells were incubated in $6 \mathrm{nmol} / \mathrm{l}$ leptin for 12 and $24 \mathrm{~h}$, the stimulation of glucose uptake by fAd and gAd was prevented, whereas $6 \mathrm{~h}$ pretreatment with the same dose of leptin had no inhibitory effect (Fig. 1c, d).

Changes of AdipoR1 and AdipoR2 expression in response to leptin We performed real-time quantitative PCR and western blotting to determine whether leptin $(60 \mathrm{nmol} / \mathrm{l}$, $24 \mathrm{~h}$ ) regulated mRNA and protein levels of the ADIPOR isoforms. It was observed that leptin treatment reduced both mRNA expression and protein levels of ADIPOR1 and ADIPOR2 compared with normal growth conditions (Fig. 1e-h). Adipor 1 mRNA expression (Fig. 1e) decreased by $\sim 45 \%$, whereas an approximate $50 \%$ reduction was observed for Adipor 2 mRNA expression (Fig. 1g). Protein levels of each isoform decreased by about $60 \%$ (Fig. 1f, h).

Effect of fAd and gAd on fatty acid uptake and regulation by leptin To examine long-chain fatty acid uptake in L6 cells, we measured $\left[9,10-{ }^{3} \mathrm{H}\right]$ palmitate uptake. This study demonstrated that both fAd and $\operatorname{gAd}(5 \mu \mathrm{g} / \mathrm{ml}, 6 \mathrm{~h})$ significantly increased palmitate uptake (Fig. 2a, b). Pretreatment of cells with leptin (60 nmol/1, $24 \mathrm{~h})$ did not alter basal fatty acid uptake levels, but did prevent the increase in palmitate uptake stimulated by fAd or gAd (Fig. 2a, b). Levels of several fatty acid transporters, including FATP1, FATP4 and CD36, were examined in response to fAd or $\mathrm{gAd}(5 \mu \mathrm{g} / \mathrm{ml}, 6 \mathrm{~h})$. CD36 levels significantly increased after fAd or gAd treatment under normal, but not under leptin treatment conditions (Fig. 2c, d). No change of FATP1 and FATP4 levels was found in response to fAd and gAd (data not shown).

Regulation of AMPK by fAd and gAd and role in stimulation of fatty acid uptake To clarify the regulation of AMPK by fAd and gAd, as well as the subsequent role of AMPK in mediating the increased fatty acid uptake, we introduced siRNA to reduce $A M P K \alpha$ expression and used compound $\mathrm{C}$, a pharmacological inhibitor, to block AMPK activity. As shown in Fig. 3a, b, compound C clearly abrogated the ability of fAd and $\operatorname{gAd}(5 \mu \mathrm{g} / \mathrm{ml}, 6 \mathrm{~h})$ to stimulate palmitate uptake. Use of siRNA lowered $A M P K \alpha 1$ and -2 expression by $70 \%$ (Fig. 3c) compared with cells transfected with a scrambled siRNA sequence; under these conditions stimulation of fatty acid uptake by fAd was attenuated (Fig. 3d). Indeed, fAd and gAd (5 $\mathrm{gg} /$ $\mathrm{ml}, 5 \mathrm{~min}$ and $15 \mathrm{~min}$ ) were found to increase phosphorylation of AMPK under normal conditions but not after leptin treatment (60 nmol/1, 24 h) (Fig. 3e, f). AICAR has been showed to increase phosphorylation of AMPK through mimicking effects of AMP by its derivative 5aminoimidazole-4-carboxamide-ribotide (AICAribotide; ZMP) [32, 33]. The phosphorylation of AMPK stimulated by AICAR was not affected by leptin treatment (Fig. 3g). We did not detect any significant effect of leptin $(60 \mathrm{nmol} / \mathrm{l}$, $24 \mathrm{~h}$ ) on total levels of AMPK $\alpha 1$ and AMPK $\alpha 2$. Phosphorylation of AMPK was not significantly altered by leptin treatment under these conditions (data not shown).

Regulation of fatty acid oxidation by fAd and gAd in the absence or presence of leptin Fatty acid oxidation was also examined in the absence or presence of leptin $(60 \mathrm{nmol} / \mathrm{l}$, $24 \mathrm{~h})$. Under normal conditions, fAd and $\mathrm{gAd}(5 \mu \mathrm{g} / \mathrm{ml}$, $6 \mathrm{~h})$ stimulated palmitate oxidation, with AICAR (2 mmol/ 1, $6 \mathrm{~h}$ ) used as a positive control in this assay (Fig. 4a, b). The ability of fAd and gAd to enhance palmitate oxidation was abolished by pretreatment of cells with leptin (Fig. 4a, b). Of note, leptin alone (60 nmol/1, $24 \mathrm{~h})$ induced a slight decrease in levels of palmitate oxidation in L6 cells. Phosphorylation of ACC was also measured under similar conditions and an increased phosphorylation of ACC (Ser79), which correlates with decreased activity of this enzyme, was observed in response to fAd and gAd $(5 \mu \mathrm{g} / \mathrm{ml}, 5 \mathrm{~min}$ and $15 \mathrm{~min}$ ) (Fig. 4c, d). However, no significant difference was 
Fig. 1 Effects of leptin on stimulation of glucose uptake by fAd and gAd, and by ADIPOR expression. Glucose uptake was measured after cells were incubated with (a) fAd ( $5 \mu \mathrm{g} / \mathrm{ml}$; black squares) or (b) gAd (5 $\mu \mathrm{g}$ / $\mathrm{ml}$; black squares) for $2 \mathrm{~h}$ under normal conditions and after leptin pretreatment $(0.3,0.6,3,6$, $60 \mathrm{nmol} / \mathrm{l}, 24 \mathrm{~h}$ ). We also examined glucose uptake in response to fAd (c) or gAd (d) after cells were incubated with leptin $(6 \mathrm{nmol} / \mathrm{l})$ for different time courses $(6,12,24 \mathrm{~h})$. White circles, control. Expression of ADIPOR1 (e, f) and ADIPOR2 $(\mathbf{g}, \mathbf{h})$ was analysed by real-time quantitative PCR and western blotting under normal and leptin treatment $(60 \mathrm{nmol} / 1,24 \mathrm{~h})$ conditions. Representative western blots are shown, with quantitative data $(\mathbf{f}, \mathbf{g})$. Values for real-time quantitative PCR and western blotting are expressed relative to $\beta$-actin as means \pm SEM of $n \geq 4 .{ }^{*} p<0.05$ with respect to control. ${ }^{\dagger} p<0.05$ with respect to adiponectin alone (i.e. no leptin pretreatment)
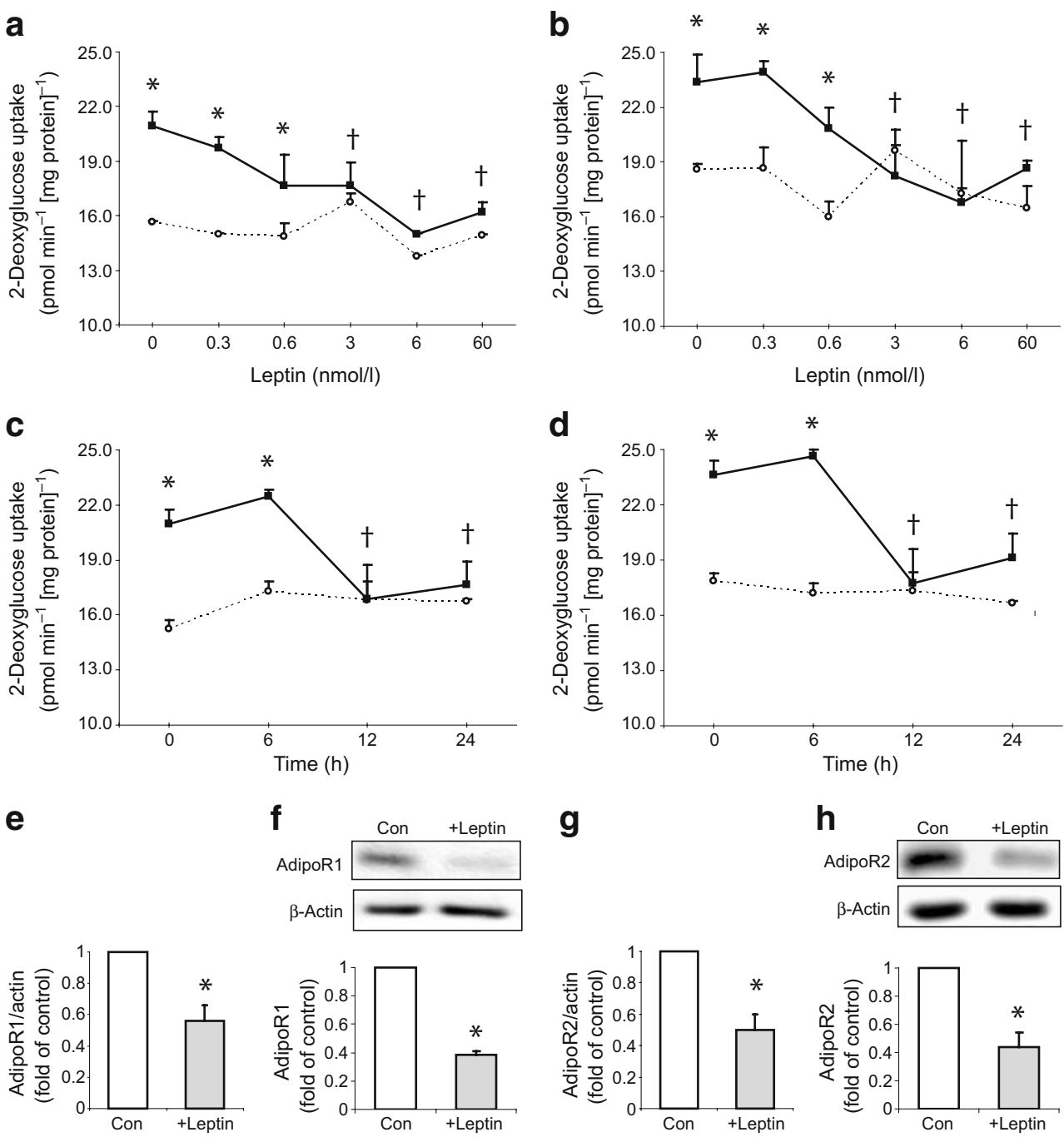

detected when cells were treated with leptin (Fig. 4c, d). Total ACC levels were unaltered by leptin (data not shown).

Effect of leptin on insulin-sensitising effects of fAd and crosstalk between adiponectin and insulin signalling L6 myotubes were pretreated with fAd $(5 \mu \mathrm{g} / \mathrm{ml}, 15 \mathrm{~min})$ then treated with insulin $(10 \mathrm{nmol} / 1,5 \mathrm{~min})$. As expected, insulin stimulated phosphorylation of IRS-1 (Tyr612) and Akt (Thr308), while no stimulation was observed in response to fAd alone (Fig. 5a). Importantly, the insulin-stimulated phosphorylation of IRS-1 and Akt was markedly enhanced in L6 cells pretreated with fAd (Fig. 5a). The adiponectinenhanced insulin signalling was blunted by leptin treatment $(60 \mathrm{nmol} / \mathrm{l}, 24 \mathrm{~h})$ with the levels of IRS-1 and Akt phosphorylation returning to those induced in the presence of insulin alone (Fig. 5a). Using immunoprecipitation of LKB1 and western blotting for APPL1, we also found that fAd $(5 \mu \mathrm{g} / \mathrm{ml}, 5 \mathrm{~min}$ and $10 \mathrm{~min})$ stimulated the interaction of LKB1 with APPL1 (Fig. 5b) and that this interaction was abolished by leptin pretreatment $(60 \mathrm{nmol} / \mathrm{l}, 24 \mathrm{~h})$. LKB1 expression was not altered by leptin ( \pm adiponectin) under the conditions used in these experiments (data not shown).

\section{Discussion}

Leptin and adiponectin are both important for the maintenance of appropriate insulin sensitivity and overall energy balance $[3,4,34]$. Changes in skeletal muscle insulin action play a central role in the development of metabolic changes in the metabolic syndrome, while, of particular relevance to this study, both leptin and adiponectin have been extensively documented to regulate basal and insulin-stimulated carbohydrate and fatty acid metabolism in skeletal muscle [35]. However, very little is known about their potential interaction in regulating metabolism in this tissue. In this 
Fig. 2 Effects of fAd and gAd on long-chain fatty acid uptake and regulation by leptin pretreatment. After treatment with or without leptin $(60 \mathrm{nmol} / 1$, $24 \mathrm{~h}$ ), palmitate uptake was measured in response to $6 \mathrm{~h}$ incubation with (a) fAd (5 $\mu \mathrm{g} /$ $\mathrm{ml})$ or $(\mathbf{b}) \operatorname{gAd}(5 \mu \mathrm{g} / \mathrm{ml})$.

Values are means \pm SEM of $n \geq 3$ c, d Expression of fatty acid transporter CD36 was examined under similar conditions for fAd and gAd respectively. Representative blots are shown, with quantitative data in bar graphs expressed as means \pm SEM of at least three independent experiments. ${ }^{*} p<0.05$ with respect to control a

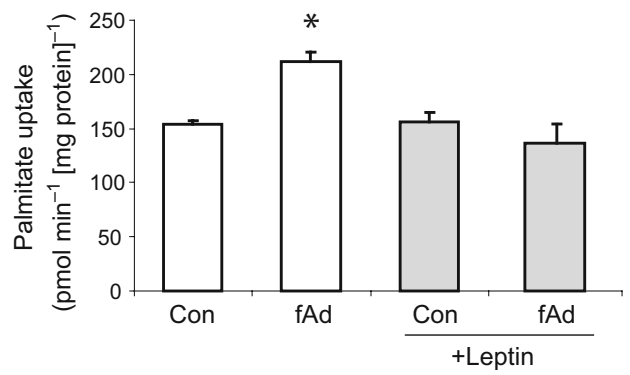

C
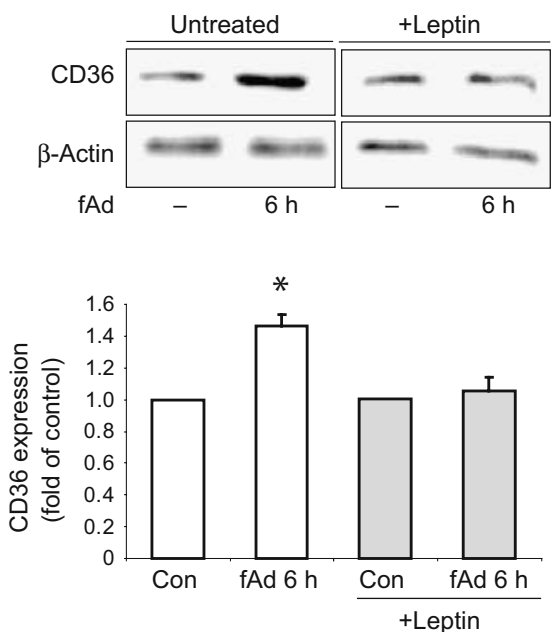

b

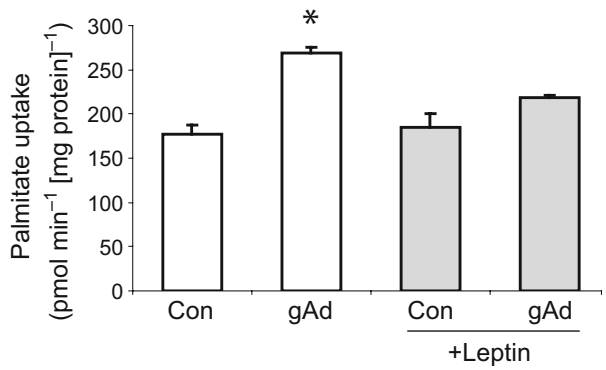

d
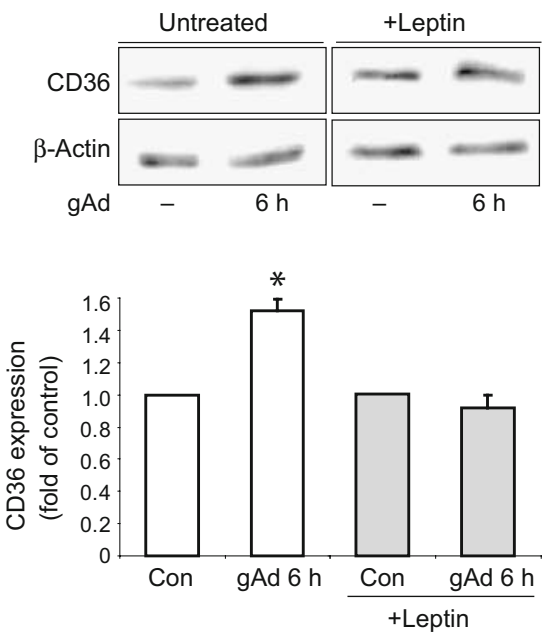

study, we investigated whether high leptin levels, a condition commonly seen in human obesity, affected the ability of adiponectin to exert its metabolic effects in skeletal muscle cells.

We have revealed that high leptin concentrations exert a direct negative effect on insulin-stimulated glucose uptake in cultured L6 skeletal muscle cells [36, 37], suggesting that hyperleptinaemia could contribute to the development of insulin resistance. However, previous work demonstrated that leptin exposure did not alter insulin-stimulated glucose uptake in isolated rodent skeletal muscle, while enhanced insulin-stimulated glucose uptake was observed in high-fat fed rats with long term leptin administration [38-41]. A difference in leptin concentration and time of exposure to leptin could account for these observations. Here, we examined the effect of chronic leptin treatment on adiponectin-stimulated glucose uptake. Consistent with previous results from ourselves and others [7, 12, 25], we found that gAd and fAd increased glucose uptake in L6 skeletal muscle cells after $2 \mathrm{~h}$ treatment. Importantly, we demonstrate for the first time that this response was attenuated in the presence of leptin in a dose- and timedependent manner. A statistically significant inhibitory effect was found at $3 \mathrm{nmol} / \mathrm{l}$ leptin and above. Although absolute leptin levels vary among normal and obese individuals, $3 \mathrm{nmol} / \mathrm{l}$ plasma leptin is in keeping with levels observed in obese individuals [42, 43]. The time-course experiment revealed that a prolonged pretreatment time (longer than $12 \mathrm{~h}$ ) is necessary for leptin to exert an inhibitory effect.

Thus, to investigate potential underlying mechanisms, we first examined changes in levels of ADIPORs, of which two isoforms, ADIPOR1 and ADIPOR2, exist in skeletal muscle. ADIPOR1 and ADIPOR2 differ with respect to tissue distribution and affinity for adiponectin forms, with ADIPOR1 exhibiting a higher binding affinity for gAd and ADIPOR2 for fAd [8]. We have also previously documented that L6 cells express ADIPOR1 in a 6:1 ratio compared with ADIPOR2 [25]. We found that mRNA and protein levels of ADIPOR1 and ADIPOR2 were decreased after exposure of cells to leptin. The most simple interpretation of this observation is that there would be a corresponding reduction in the availability of these receptors to bind adiponectin and a subsequent decline in gAdand fAd-stimulated effects. Many, but not all, studies in animal models [44] and humans [13, 14] have also suggested an important role for alterations in ADIPORs in the pathogenesis of insulin resistance and diabetes. Of particular interest, Adipor $1^{-/-}$mice showed increased 
Fig. 3 Role of AMPK in adiponectin-stimulated fatty acid uptake. We examined fatty acid uptake in response to (a) fAd or (b) $\operatorname{gAd}(5 \mu \mathrm{g} / \mathrm{ml}, 6 \mathrm{~h})$ in the presence or absence of the AMPK inhibitor compound C $(20 \mu \mathrm{mol} / 1,30 \mathrm{~min}$ pretreatment). c Two $\alpha$ subunits of $A M P K(A M P K \alpha 1 / 2)$ were silenced by combination of two siRNA sequences. Fatty acid uptake was then examined after incubation with fAd (d). The degree of phosphorylation of AMPK was measured after 5 and 15 min incubation with $5 \mu \mathrm{g} / \mathrm{ml} \mathrm{fAd} \mathrm{(e)} \mathrm{or} \mathrm{gAd} \mathrm{(f)} \mathrm{in}$ control (Con) and leptin-treated $(60 \mathrm{nmol} / \mathrm{l}, 24 \mathrm{~h})$ cells. $\mathrm{g}$ AICAR-stimulated phosphorylation of AMPK was measured under similar conditions. Representative western blots are shown, with quantitative data expressed as means \pm SEM of at least three independent experiments. ${ }^{*} p<0.05$ with respect to control
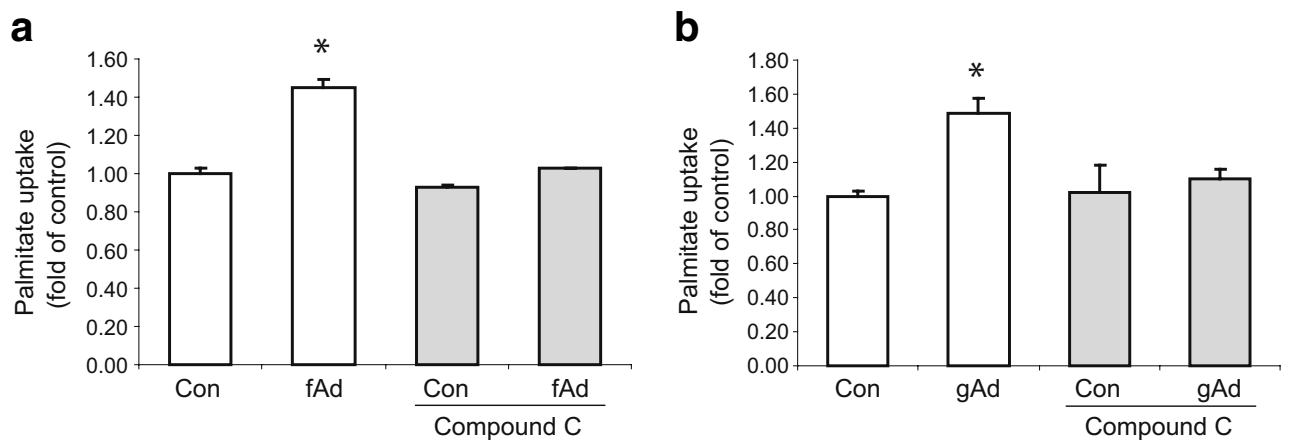

C
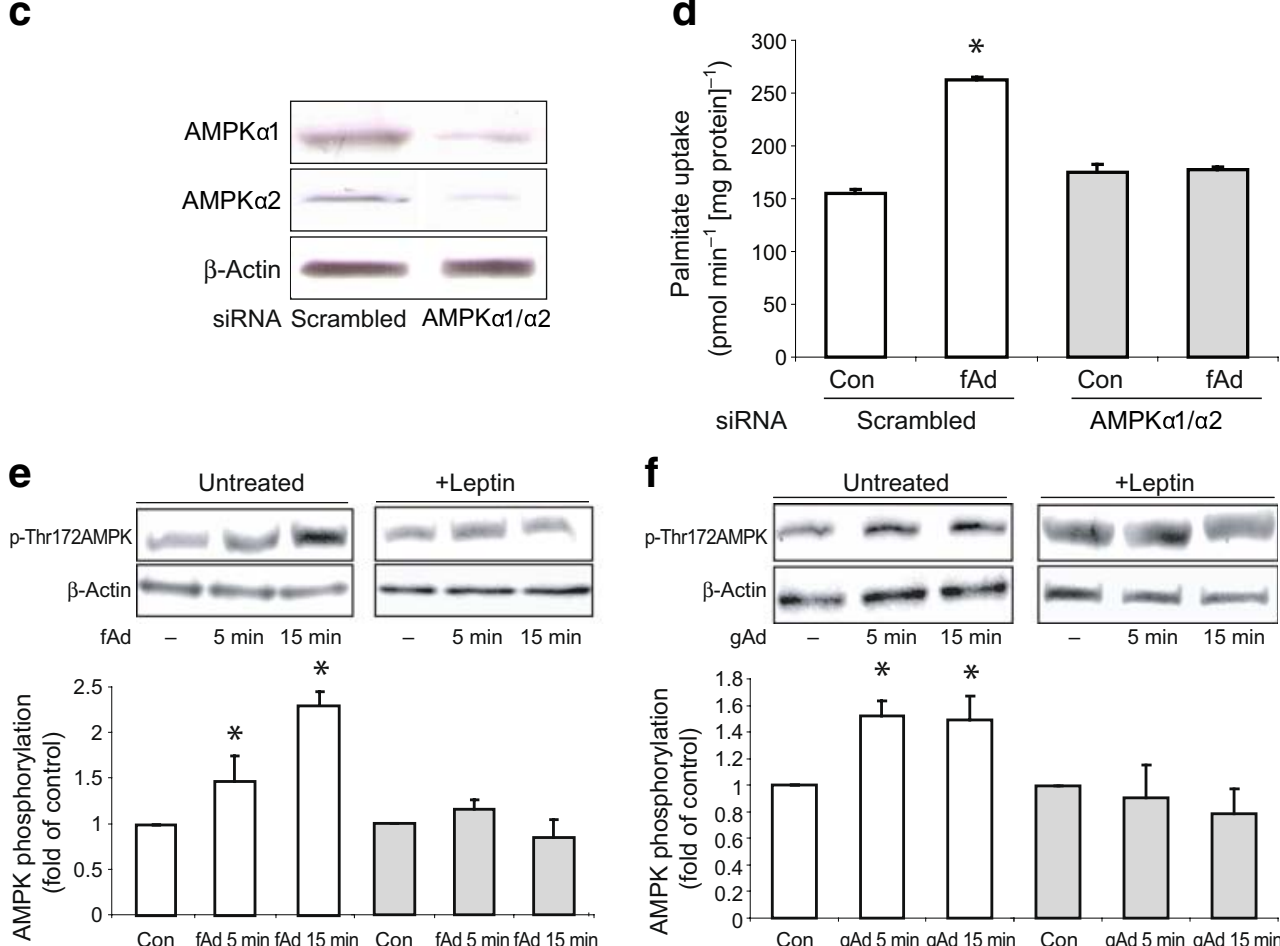

f Untreated
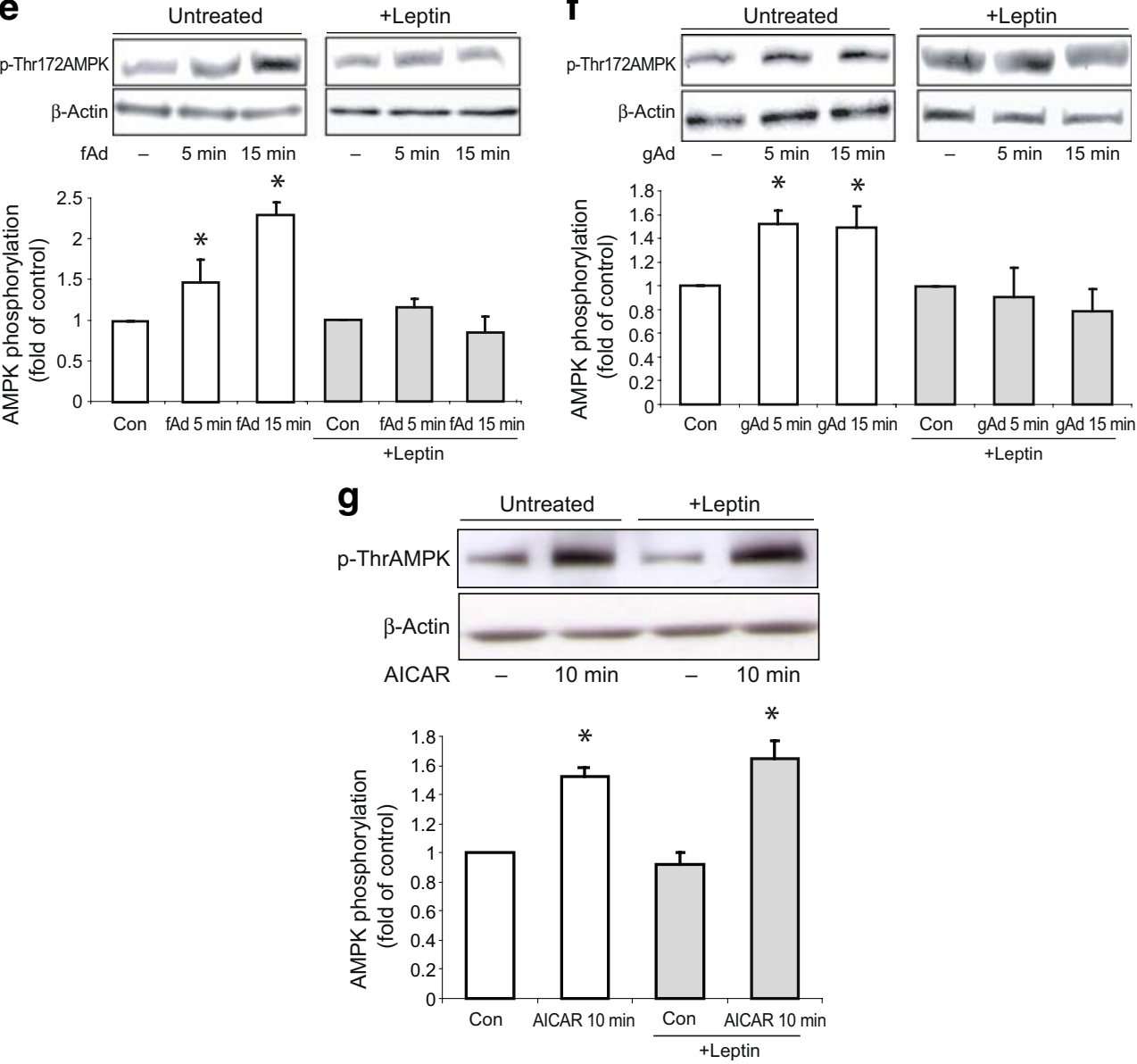
Fig. 4 Regulation of effects of fAd and gAd on fatty acid oxidation and on ACC phosphorylation by leptin. We examined palmitate oxidation in response to fAd (a) or gAd (b) $(5 \mu \mathrm{g} / \mathrm{ml}, 6 \mathrm{~h})$ with or without leptin pretreatment $(60 \mathrm{nmol} / \mathrm{l}$, $24 \mathrm{~h}) . \operatorname{AICAR}(2 \mathrm{mmol} / \mathrm{l}, 6 \mathrm{~h})$ was used as positive control in this assay. c, $\mathbf{d}$ The degree of phosphorylation of ACC was measured after 5 and 15 min incubation with $5 \mu \mathrm{g} / \mathrm{ml}$ fAd or gAd respectively in control and leptin-treated (60 nmol/1, $24 \mathrm{~h})$ cells. Representative western blots are shown, with quantitative data in bar graphs, as means \pm SEM of at least three independent experiments. ${ }^{*} p<0.05$ with respect to control. CPMA, radioactivity counts per min
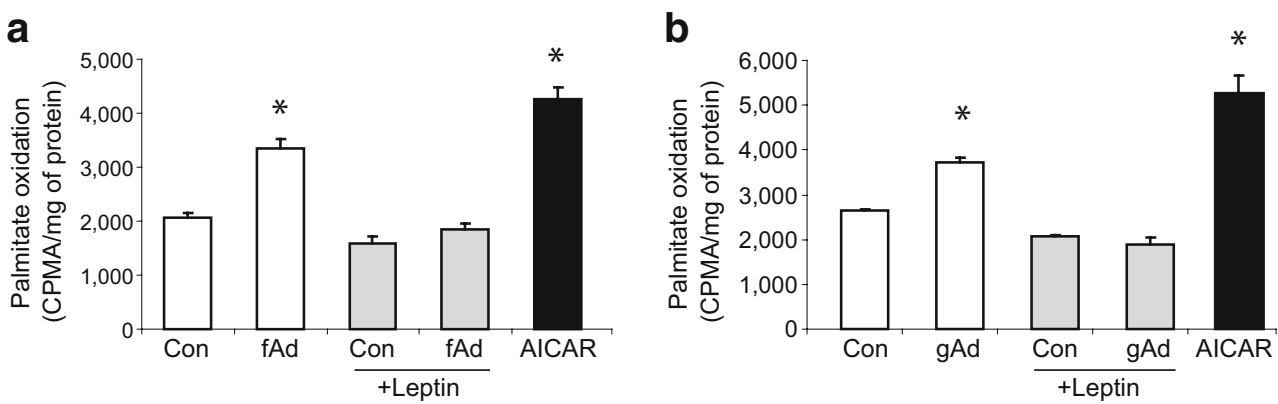

C
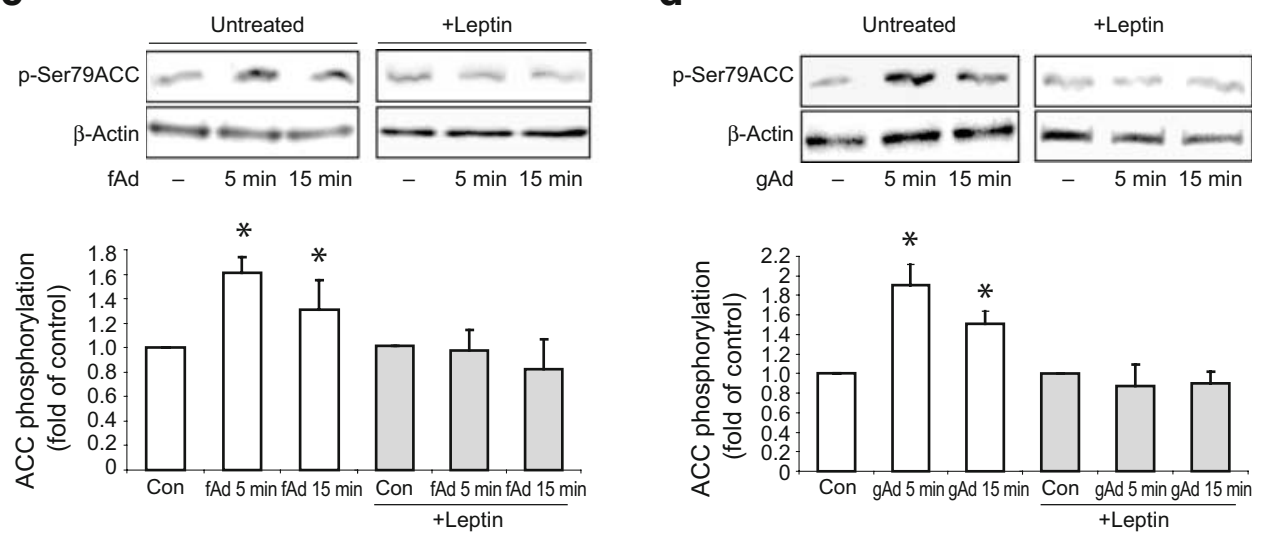

adiposity associated with decreased glucose tolerance and energy expenditure, whereas Adipor $2^{-/-}$mice were lean and resistant to high-fat diet-induced obesity [44]. In human studies, Steinberg's group has shown that skeletal muscle from obese or obese type 2 diabetic patients showed decreased sensitivity to gAd, yet ADIPOR 1 levels were higher, suggesting that aspects downstream of the receptor may inhibit adiponectin action [13]. In contrast to our current study, others also found that leptin $(2.5 \mu \mathrm{g} / \mathrm{ml}, 6 \mathrm{~h})$ stimulated ADIPOR 1 expression in myotubes derived from lean healthy individuals, but not in those derived from obese or obese diabetic patients [45]. Finally, exercise is a well-known effective therapeutic approach for treatment of diabetes, with current literature suggesting that although exercise does not tend to alter circulating adiponectin levels, there may be a correlation with increased skeletal muscle ADIPOR in exercised animals or humans [15]. Leptin, therefore, clearly has a capacity to regulate ADIPOR expression and under certain circumstances this may be of physiological importance.

A number of studies have indicated diminished fatty acid oxidation in skeletal muscle of obese and type 2 diabetic patients $[46,47]$. The impaired capability of skeletal muscle to appropriately oxidise fatty acids may be associated with development of insulin resistance. Leptin has been shown to acutely stimulate fatty acid oxidation in $\mathrm{C} 2 \mathrm{C} 12$ cells and primary human skeletal muscle cells [48, 49]. Here, we found that chronic leptin treatment had no effect on the basal fatty acid oxidation rate, which is consistent with a previous publication demonstrating no change of fatty acid oxidation in resting soleus muscle in response to chronic leptin administration [41]. Adiponectin has also been shown to mediate insulin-sensitising effects and one potential mechanism is the enhancement of skeletal muscle fatty acid uptake and oxidation, thus avoiding deleterious lipotoxicity [50]. We have previously shown that adiponectin stimulated fatty acid uptake in skeletal muscle cells [25]. Here, we provide evidence that the mechanism of increased fatty acid uptake after $6 \mathrm{~h}$ fAd or gAd treatment may involve increased expression of CD36. Furthermore, and as expected, we show that fAd and gAd phosphorylate AMPK at 5 and $15 \mathrm{~min}$. For the first time, we also show that fAd- and gAd-stimulated fatty acid uptake is mediated via AMPK. Similar observations on the important role of AMPK in fatty acid transporter expression have been reported in cardiac myocytes and mouse skeletal muscle [51, 52]. Importantly, all of the above were attenuated when cells were pretreated with leptin. Although transient activation of AMPK was induced by leptin in skeletal muscle in vitro, there was no significant change in phosphorylation or total levels of AMPK after cells were treated with leptin for $24 \mathrm{~h}$, indicating that lack of adiponectin action under these conditions was not simply due to an already elevated level of AMPK phosphorylation. 


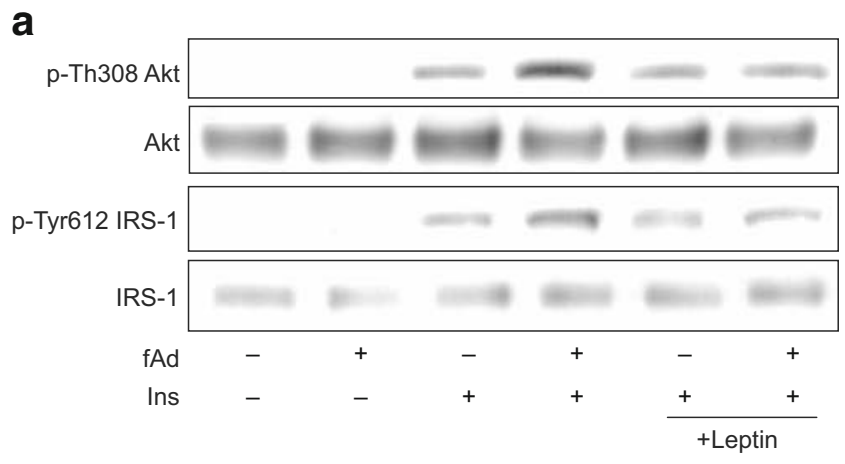

b
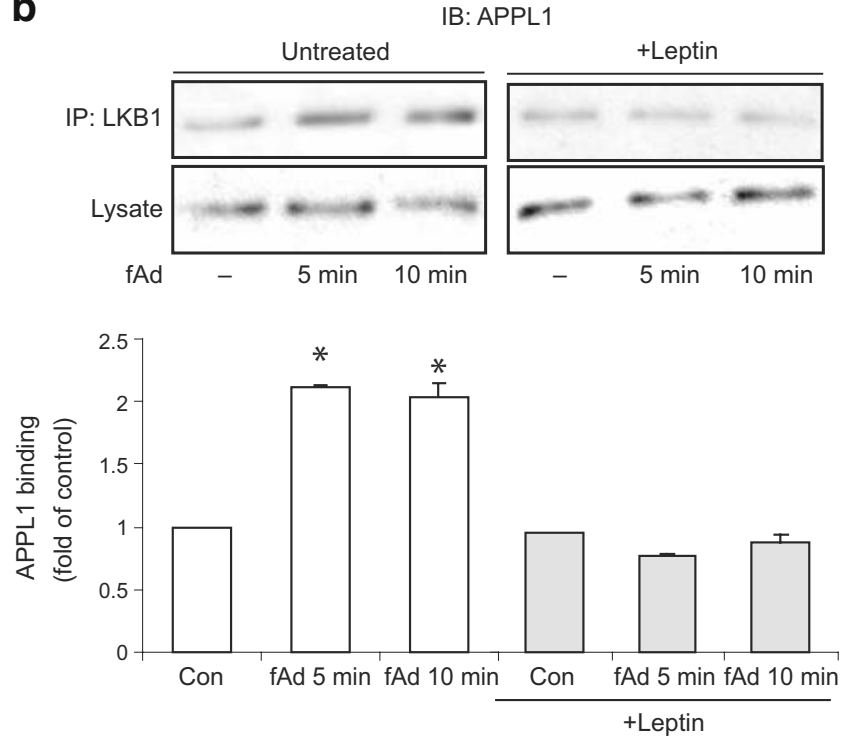

Fig. 5 Molecular mechanisms mediating crosstalk between adiponectin and insulin signalling in the absence or presence of leptin. a Phosphorylation and total protein levels of Akt and IRS-1 were detected by western blot (representative blot shown) after cells were treated with or without fAd $(5 \mu \mathrm{g} / \mathrm{ml})$ for $15 \mathrm{~min}$, followed by incubation with insulin $(10 \mathrm{nmol} / 1,5 \mathrm{~min})$ under normal and leptin pretreated $(60 \mathrm{nmol} / \mathrm{l}, 24 \mathrm{~h})$ conditions. b Presence of APPL1 in LKB1 immunoprecipitates (IP) from cells treated with fAd $(5 \mu \mathrm{g} / \mathrm{ml})$ for 5 or 10 min was measured by western blotting under normal and leptin pretreated $(60 \mathrm{nmol} / 1,24 \mathrm{~h})$ conditions. Representative western blots are shown, with quantitative analysis in bar graph, as means \pm SEM of at least three independent experiments. ${ }^{*} p<0.05$ with respect to control

Furthermore, AICAR-stimulated phosphorylation of AMPK was not affected by leptin at concentrations and times that inhibit adiponectin action, indicating the inhibitory effect of leptin on adiponectin is not non-specific and occurs upstream of AMPK. Our results also indicate that gAd and fAd stimulated ACC phosphorylation, causing inhibition of this enzyme and subsequent increases in palmitate oxidation, all of which were attenuated by leptin. Thus, it is clear from our in vitro studies that the normally beneficial effects of adiponectin leading to enhanced fatty acid uptake and oxidation are lost in the presence of high leptin levels and that this is conceivably of great pathophysiological significance in the development of insulin resistance in individuals with hyperleptinaemia and normal skeletal muscle leptin sensitivity.

Recent evidence has also indicated that adiponectin can improve insulin sensitivity via acute effects involving intracellular signalling pathway crosstalk. Initial studies identified APPL1 as a novel binding partner of AdipoRs in response to adiponectin stimulation and established a role for this protein in mediating nitric oxide production in endothelial cells [16] or in crosstalk with insulin signalling in $\mathrm{C} 2 \mathrm{C} 12$ muscle cells [17]. Subsequent work demonstrated that the APPL1/LKB1/AMPK axis plays a vital role in mediating the insulin-sensitising effects of adiponectin [9], and most recently it was shown that APPL1 can release Akt trapped in a cytosolic localisation by its inhibitor TRB3, thereby promoting Akt activation by insulin [18]. Here, we demonstrate a significant synergistic effect on stimulation of IRS-1 (Y612) and Akt (T308) when cells were pretreated with fAd for 15 min followed by 5 min insulin treatment. Interestingly, pretreatment of cells with high leptin levels attenuated the insulin-sensitising function of fAd. Since we have shown that leptin attenuated adiponectin-stimulated AMPK phosphorylation and it has been shown that adiponectin sensitises insulin signalling via an LKB1/ AMPK-dependent mechanism [9], we propose that blunting of adiponectin-stimulated AMPK phosphoryation by leptin and the synergistic effects of adiponectin on insulin signalling occur through inhibition of LKB1/AMPK activation. Therefore, we employed a co-immunoprecipitation approach to study the interaction of LKB1 with several signalling molecules potentially involved in adiponectininsulin crosstalk. We saw that under normal conditions fAd increased binding of LKB1 to the upstream regulator APPL1 and that this interaction was blunted by the presence of leptin. Our data suggest that leptin inhibits AMPK stimulation by adiponectin probably via inhibited interaction between LKB1 and APPL1.

In summary, although it is well established that circulating adiponectin levels, particularly high molecular weight adiponectin, decrease in obesity, there is currently an increasing awareness of the potential development of adiponectin resistance in individuals exhibiting features of the metabolic syndrome [53]. Taken together, our data demonstrate that prolonged treatment of skeletal muscle cells with elevated leptin concentrations reduced adiponectin signalling and metabolic effects in skeletal muscle cells. Hence, our study indicates crosstalk between leptin and adiponectin signalling and action, and suggests that hyperleptinaemia may be an important contributory factor leading to adiponectin resistance in skeletal muscle, thus exacerbating the disturbed metabolic milieu. 
Acknowledgements Funding for this work was provided by The Canadian Institutes of Health Research (CIHR) via an operating grant and a New Investigator award to G. Sweeney. X. Fang acknowledges Doctoral Studentship support from The Heart and Stroke Foundation of Canada and The Canadian Diabetes Association.

Duality of interest The authors declare that there is no duality of interest associated with this manuscript.

\section{References}

1. Abel ED, Litwin SE, Sweeney G (2008) Cardiac remodeling in obesity. Physiol Rev 88:389-419

2. Kahn SE, Hull RL, Utzschneider KM (2006) Mechanisms linking obesity to insulin resistance and type 2 diabetes. Nature 444:840-846

3. Badman MK, Flier JS (2007) The adipocyte as an active participant in energy balance and metabolism. Gastroenterology 132:2103-2115

4. Fang X, Sweeney G (2006) Mechanisms regulating energy metabolism by adiponectin in obesity and diabetes. Biochem Soc Trans 34:798-801

5. Kadowaki T, Yamauchi T, Kubota N (2008) The physiological and pathophysiological role of adiponectin and adiponectin receptors in the peripheral tissues and CNS. FEBS Lett 582:74-80

6. Berg AH, Combs TP, Du X, Brownlee M, Scherer PE (2001) The adipocyte-secreted protein Acrp30 enhances hepatic insulin action. Nat Med 7:947-953

7. Ceddia RB, Somwar R, Maida A, Fang X, Bikopoulos G, Sweeney G (2005) Globular adiponectin increases GLUT4 translocation and glucose uptake but reduces glycogen synthesis in rat skeletal muscle cells. Diabetologia 48:132-139

8. Yamauchi T, Kamon J, Ito Y et al (2003) Cloning of adiponectin receptors that mediate antidiabetic metabolic effects. Nature 423:762-769

9. Wang C, Mao X, Wang L et al (2007) Adiponectin sensitizes insulin signaling by reducing p70 S6 kinase-mediated serine phosphorylation of IRS-1. J Biol Chem 282:7991-7996

10. Xu A, Chan KW, Hoo RL et al (2005) Testosterone selectively reduces the high molecular weight form of adiponectin by inhibiting its secretion from adipocytes. J Biol Chem 280:18073-18080

11. Tsao TS, Murrey HE, Hug C, Lee DH, Lodish HF (2002) Oligomerization state-dependent activation of NF-kappa B signaling pathway by adipocyte complement-related protein of $30 \mathrm{kDa}$ (Acrp30). J Biol Chem 277:29359-29362

12. Fruebis J, Tsao TS, Javorschi S et al (2001) Proteolytic cleavage product of $30-\mathrm{kDa}$ adipocyte complement-related protein increases fatty acid oxidation in muscle and causes weight loss in mice. Proc Natl Acad Sci U S A 98:2005-2010

13. Chen MB, McAinch AJ, Macaulay SL et al (2005) Impaired activation of AMP-kinase and fatty acid oxidation by globular adiponectin in cultured human skeletal muscle of obese type 2 diabetics. J Clin Endocrinol Metab 90:3665-3672

14. Liu Y, Michael MD, Kash S et al (2007) Deficiency of adiponectin receptor 2 reduces diet-induced insulin resistance but promotes type 2 diabetes. Endocrinology 148:683-692

15. Vu V, Riddell MC, Sweeney G (2007) Circulating adiponectin and adiponectin receptor expression in skeletal muscle: effects of exercise. Diabetes Metab Res Rev 23:600-611

16. Cheng KK, Lam KS, Wang Y et al (2007) Adiponectin-induced endothelial nitric oxide synthase activation and nitric oxide production are mediated by APPL1 in endothelial cells. Diabetes 56:1387-1394

17. Mao X, Kikani CK, Riojas RA et al (2006) APPL1 binds to adiponectin receptors and mediates adiponectin signalling and function. Nat Cell Biol 8:516-523
18. Cheng K, Iglesias M, Lam K et al (2009) APPL1 potentiates insulin-mediated suppression of hepatic gluconeogenesis and alleviates diabetes by counteracting the Akt inhibitor tribble-3 in mice. Cell Metabolism 9:417-427

19. Kim JY, van de Wall E, Laplante M et al (2007) Obesityassociated improvements in metabolic profile through expansion of adipose tissue. J Clin Invest 117:2621-2637

20. Sennello JA, Fayad R, Pini M, Gove ME, Fantuzzi G (2006) Transplantation of wild-type white adipose tissue normalizes metabolic, immune and inflammatory alterations in leptin-deficient ob/ob mice. Cytokine 36:261-266

21. Sweeney G (2002) Leptin signalling. Cell Signal 14:655-663

22. Mistry T, Digby JE, Desai KM, Randeva HS (2008) Leptin and adiponectin interact in the regulation of prostate cancer cell growth via modulation of p53 and bcl-2 expression. BJU Int 101:1317-1322

23. Fenton JI, Birmingham JM, Hursting SD, Hord NG (2008) Adiponectin blocks multiple signaling cascades associated with leptin-induced cell proliferation in Apc Min/+colon epithelial cells. Int J Cancer 122:2437-2445

24. Ogunwobi OO, Beales IL (2008) Globular adiponectin, acting via adiponectin receptor-1, inhibits leptin-stimulated oesophageal adenocarcinoma cell proliferation. Mol Cell Endocrinol 285:43-50

25. Fang X, Palanivel R, Zhou X et al (2005) Hyperglycemia- and hyperinsulinemia-induced alteration of adiponectin receptor expression and adiponectin effects in L6 myoblasts. J Mol Endocrinol 35:465-476

26. Wang Y, Xu A, Knight C, Xu LY, Cooper GJ (2002) Hydroxylation and glycosylation of the four conserved lysine residues in the collagenous domain of adiponectin. Potential role in the modulation of its insulin-sensitizing activity. J Biol Chem 277:19521-19529

27. Bastie CC, Hajri T, Drover VA, Grimaldi PA, Abumrad NA (2004) CD36 in myocytes channels fatty acids to a lipaseaccessible triglyceride pool that is related to cell lipid and insulin responsiveness. Diabetes 53:2209-2216

28. Yoon MJ, Lee GY, Chung JJ, Ahn YH, Hong SH, Kim JB (2006) Adiponectin increases fatty acid oxidation in skeletal muscle cells by sequential activation of AMP-activated protein kinase, p38 mitogen-activated protein kinase, and peroxisome proliferatoractivated receptor alpha. Diabetes 55:2562-2570

29. Palanivel R, Fang X, Park M et al (2007) Globular and full-length forms of adiponectin mediate specific changes in glucose and fatty acid uptake and metabolism in cardiomyocytes. Cardiovasc Res 75:148-157

30. Ceci R, Sabatini S, Duranti G, Savini I, Avigliano L, Rossi A (2007) Acute, but not chronic, leptin treatment induces acyl-CoA oxidase in C2C12 myotubes. Eur J Nutr 46:364-368

31. Palanivel R, Eguchi M, Shuralyova I, Coe I, Sweeney G (2006) Distinct effects of short-and long-term leptin treatment on glucose and fatty acid uptake and metabolism in HL-1 cardiomyocytes. Metabolism 55:1067-1075

32. Corton JM, Gillespie JG, Hawley SA, Hardie DG (1995) 5Aminoimidazole-4-carboxamide ribonucleoside. A specific method for activating AMP-activated protein kinase in intact cells? Eur J Biochem 229:558-565

33. Merrill GF, Kurth EJ, Hardie DG, Winder WW (1997) AICA riboside increases AMP-activated protein kinase, fatty acid oxidation, and glucose uptake in rat muscle. Am J Physiol 273:E1107-E1112

34. Ceddia RB, Koistinen HA, Zierath JR, Sweeney G (2002) Analysis of paradoxical observations on the association between leptin and insulin resistance. FASEB J 16:1163-1176

35. Dyck DJ, Heigenhauser GJ, Bruce CR (2006) The role of adipokines as regulators of skeletal muscle fatty acid metabolism and insulin sensitivity. Acta Physiol (Oxf) 186:5-16

36. Bates SH, Gardiner JV, Jones RB, Bloom SR, Bailey CJ (2002) Acute stimulation of glucose uptake by leptin in 16 muscle cells. Horm Metab Res 34:111-115 
37. Sweeney G, Keen J, Somwar R, Konrad D, Garg R, Klip A (2001) High leptin levels acutely inhibit insulin-stimulated glucose uptake without affecting glucose transporter 4 translocation in 16 rat skeletal muscle cells. Endocrinology 142:4806-4812

38. Ceddia RB, William WN Jr, Curi R (1999) Comparing effects of leptin and insulin on glucose metabolism in skeletal muscle: evidence for an effect of leptin on glucose uptake and decarboxylation. Int J Obes Relat Metab Disord 23:75-82

39. Harris RB (1998) Acute and chronic effects of leptin on glucose utilization in lean mice. Biochem Biophys Res Commun 245:502-509

40. Ceddia RB, William WN Jr, Curi R (1998) Leptin increases glucose transport and utilization in skeletal muscle in vitro. Gen Pharmacol 31:799-801

41. Yaspelkis BB 3rd, Singh MK, Krisan AD et al (2004) Chronic leptin treatment enhances insulin-stimulated glucose disposal in skeletal muscle of high-fat fed rodents. Life Sci 74:1801-1816

42. Considine RV, Sinha MK, Heiman ML et al (1996) Serum immunoreactive-leptin concentrations in normal-weight and obese humans. N Engl J Med 334:292-295

43. Maffei M, Halaas J, Ravussin E et al (1995) Leptin levels in human and rodent: measurement of plasma leptin and ob RNA in obese and weight-reduced subjects. Nat Med 1:1155-1161

44. Bjursell M, Ahnmark A, Bohlooly YM et al (2007) Opposing effects of adiponectin receptors 1 and 2 on energy metabolism. Diabetes 56:583-593

45. McAinch AJ, Steinberg GR, Mollica J et al (2006) Differential regulation of adiponectin receptor gene expression by adiponectin and leptin in myotubes derived from obese and diabetic individuals. Obesity (Silver Spring) 14:1898-1904
46. Kelley DE, Goodpaster B, Wing RR, Simoneau JA (1999) Skeletal muscle fatty acid metabolism in association with insulin resistance, obesity, and weight loss. Am J Physiol 277:E1130 E1141

47. Kim JY, Hickner RC, Cortright RL, Dohm GL, Houmard JA (2000) Lipid oxidation is reduced in obese human skeletal muscle. Am J Physiol Endocrinol Metab 279:E1039-E1044

48. Steinberg GR, McAinch AJ, Chen MB et al (2006) The suppressor of cytokine signaling 3 inhibits leptin activation of AMP-kinase in cultured skeletal muscle of obese humans. J Clin Endocrinol Metab 91:3592-3597

49. Suzuki A, Okamoto S, Lee S, Saito K, Shiuchi T, Minokoshi Y (2007) Leptin stimulates fatty acid oxidation and peroxisome proliferator-activated receptor alpha gene expression in mouse $\mathrm{C} 2 \mathrm{C} 12$ myoblasts by changing the subcellular localization of the alpha2 form of AMP-activated protein kinase. Mol Cell Biol $27: 4317-4327$

50. Schaffer JE (2003) Lipotoxicity: when tissues overeat. Curr Opin Lipidol 14:281-287

51. Chabowski A, Momken I, Coort SL et al (2006) Prolonged AMPK activation increases the expression of fatty acid transporters in cardiac myocytes and perfused hearts. Mol Cell Biochem 288:201-212

52. Long YC, Barnes BR, Mahlapuu M et al (2005) Role of AMPactivated protein kinase in the coordinated expression of genes controlling glucose and lipid metabolism in mouse white skeletal muscle. Diabetologia 48:2354-2364

53. Kintscher U (2007) Does adiponectin resistance exist in chronic heart failure? Eur Heart J 28:1676-1677 